\title{
Use of the Chick Embryo Model in Uveal Melanoma
}

\author{
Helen Kalirai ${ }^{a} \quad$ Haleh Shahidipour ${ }^{a}$ Sarah E. Coupland ${ }^{a}$ \\ Gregorius P.M. Luyten ${ }^{b}$ \\ a Department of Molecular and Clinical Cancer Medicine, Institute of Translational Research, \\ University of Liverpool, Liverpool, UK; ${ }^{b}$ Department of Ophthalmology, Leiden University \\ Medical Center, Leiden, The Netherlands
}

\section{Key Words}

Chick embryo $\cdot$ Uveal melanoma $\cdot$ Chorioallantoic membrane $\cdot$ Metastasis

\begin{abstract}
Animal models play a crucial role in basic and translational oncology research. Conventional rodent experiments, however, face ethical, practical and technical issues that limit their use. The chick embryo represents an accessible and economical in vivo model, which has long been used in developmental biology and for the study of angiogenesis. It is also a recognised xenograft model, and because of its lack of immune system in early development, the chick embryo has established itself as a key model system for cancer research, with which to study various steps in the metastatic process. In this chapter, we review the chick embryo model and the technical approaches adopted by cancer biologists, including advances in real-time imaging, and discuss how this has been or can be applied to improve our understanding of the biological events during uveal melanoma development and metastasis.
\end{abstract}

(c) 2015 S. Karger AG, Basel

\section{Introduction}

Investigations into the molecular and cellular processes underlying uveal melanoma development and metastasis are essential to develop therapeutic approaches directed against disseminated disease. To this end, extensive efforts have been devoted to the development of innovative and clinically relevant in vitro and in vivo preclinical models that reproduce various aspects of these processes. One such in vivo model system, first described more than a century ago for heterologous tumour growth $[1,2]$, is the chick embryo. The tissue composition and accessibility of the chick embryo chorioallantoic membrane (CAM) for experi- 
Kalirai et al.: Use of the Chick Embryo Model in Uveal Melanoma

mental interventions, which enable the fate of grafted tumour cells to be visualised and followed, has facilitated two experimental approaches in this model system. (1) Tumour cells are inoculated directly onto the highly vascularised CAM, which then sustains rapid tumour formation within several days following cell grafting. The CAM also serves as a repository of aggressive tumour cells that have the capacity to escape from the primary tumour and intravasate into the host vasculature. This spontaneous metastasis setting provides a unique system with which to study tumour cell dissemination in vivo. (2) Tumour cells are inoculated intravenously and their ability to extravasate the capillary bed and colonise internal organs is studied. A validation of this model system in uveal melanoma is essential to enhance the research resources available with which to study the steps that cells from the primary tumour must take to establish metastases at distant sites in the body.

\section{The Chick Embryo and CAM Development}

The various stages of chick embryo development, as classified in 1951 by Hamburger and Hamilton [3], have enabled researchers to take advantage of this in vivo system for biomedical research.

In the chick embryo, the chorioallantois is formed between days 4 and 5 of development, when the outer mesodermal layer of the allantois fuses with the mesodermal lining of the chorion, and a network of blood vessels is gradually formed between the two layers. The CAM serves as the lungs of the embryo and fully envelops it by day 12 of incubation. Histologically, the CAM contains three major layers: (1) the ectoderm attached to the shell membrane, (2) the mesoderm enriched in blood vessels and stromal components, and (3) the endoderm facing the allantoic cavity. The three layers form a thin transparent collagenous matrix approximately $100 \mu \mathrm{m}$ in depth. By day 10 of incubation, the CAM also comprises the fully developed ectoderm capillary plexus, which represents a network of tiny capillaries connecting the arterial and venous blood vessel networks. Thus, until day 11 or 12 of chick embryo development, the developing CAM vasculature is highly angiogenic and ready to form new branching vessels in response to additional pro-angiogenic stimuli.

CAM arterioles and venules are accompanied by a pair of interconnected lymphatics. Chick lymphatic formation occurs after the initial angiogenesis is well underway and the chick embryo immune system does not begin to function until around two weeks into its development. Evidence of differentiated immune cells indicates heterophil and monocyte formation detected around embryonic day (E) 10-15.

Internal organs develop between E1 and E7, with haemopoieis commencing in the liver and formation of the ciliary body in the eye at E7. More detailed information about chick embryo development, staging and anatomy are beyond the scope of this review but are provided in Bellairs and Osmond [4].

The developing chick embryo is a self-contained and self-sufficient entity undergoing normal development at $37^{\circ} \mathrm{C}$ and $60 \%$ humidity. This ensures consistent viability of animals without artificial support media, special culture requirements or storage facilities. Chicken eggs are available all year round, are inexpensive and can be incubated to any developmental stage of interest, simplifying experimental design. Moreover, the accessibility of the in ovo chick and the relative transparency of the internal structures makes it readily adaptable to complex investigative work with continued observation. Researchers have taken advantage of these characteristics to firmly establish the chick embryo as a biological model for many disease pathologies. Indeed, the highly vascularised CAM promotes the efficiency of tumour cell grafting and is one of the earliest models used to grow tumour xenografts. In 1911, Rous and Murphy [5] demonstrated the growth of the Rous 45 chicken sarcoma transplanted onto 
the CAM. One year later, Murphy [6] reported that mouse and rat tumours implanted onto the CAM could be maintained by continuous passage from egg to egg. Today, the chick embryo is an established biological platform for human carcinogenesis, enabling researchers to study tumour mass formation, tumour-induced angiogenesis, intra- and extravasation of tumour cells and metastatic colonisation of distant organs [for reviews, see 7-10].

\section{The Chick Embryo Model in Cancer Biology}

The chick embryo as a model to study tumour metastasis has been reported in a variety of cancer types including glioma [11], prostate [12], colorectal [13], renal [14], ovarian [15] and small-cell lung cancer [16]. In uveal melanoma, a small number of groups including our own have begun to examine the suitability of this model to monitor the growth and metastatic properties of uveal melanoma cells either grafted onto the CAM, injected intravenously or transplanted into the optic cup. Luyten et al. [17] were the first to describe such studies using uveal melanoma cells cultivated from the subcutaneous metastatic lesion of a 46-year-old patient who had undergone enucleation for uveal melanoma some 28 years earlier. At E3, $10^{2}-10^{3}$ metastatic uveal melanoma cells were introduced into the developing eye of twenty embryos by microinjection. At E19, four of eighteen surviving embryos showed intraocular tumour growth in the eyes that had developed normally. Metastatic uveal melanoma cells were observed adherent to the ciliary body and along the hyaloid artery and tunica vasculosa lentis, as confirmed immunohistochemically using antibodies against S100 and HMB45, but were not reported in the choroid as was observed for human skin melanoma cells injected into the optic cup in the same study. The ability of skin-derived melanoma cells to form tumours in the choroid of the chick embryo optic cup has more recently been confirmed using the B16-F1 melanoma cell line transplanted behind the lens on E3.5 and grown for 3 days [18]. The malignant growth of these cells at this site was also enhanced by bone morphogenetic protein-2 providing a rationale for further orthotopic studies using a panel of well-characterised uveal melanoma cell lines.

A more common approach used to study tumour biology in the chick embryo model is the so-called 'CAM assay'. In this assay, tumour cells are grafted onto the CAM either in the form of tumour explants or tumour cell suspensions. To achieve this, a small window is made in the shell above the 'lowered' CAM. The CAM is lowered at E3-E4 by removing a small volume (approx. $3 \mathrm{ml}$ ) of albumen with a 5-ml syringe and 18-gauge needle. By extracting the albumen, the CAM of the fertilised egg is separated from the top part of the shell, which allows for a small hinged window to be cut in the shell of the egg, without damaging the embryonic structures. The shell window is then sealed with tape and the egg is placed back in the incubator until E7 (fig. 1). We have conducted preliminary CAM studies using two uveal melanoma cell lines to date, OMM1 [19] and 92.1 [20], labelled with green fluorescent protein (GFP) to enhance visualisation. Prior to inoculation, the junction of two or more large blood vessels was selected as the graft site. One of the vessels was then 'traumatised' using a pipette tip to induce a small bleed, a technique that has previously been used to enhance engraftment of the tumour cells [11]. A 'wet pellet' of $2 \times 10^{6}$ uveal melanoma cells was then introduced onto this site at E7. After 7 days, we observed tumour nodules for both the OMM1 and 92.1 cells of up to $1 \mathrm{~mm}^{3}$, with a tumour nodule-forming efficiency in the surviving embryos of 10 and $50 \%$, respectively [Shahidipour et al., unpubl. data]. The OMM1 tumour nodules also demonstrated a capacity to invade the chorionic epithelium forming a visible tumour mass on the chick embryo side, i.e. underneath the CAM, with an overlying network of blood vessels. The formation of tumour nodules on the underside of the CAM has also been reported for smallcell lung cancer cells [16]. Tumour nodules formed on the CAM were then dissected from the 
Ocular Oncology

and Pathology
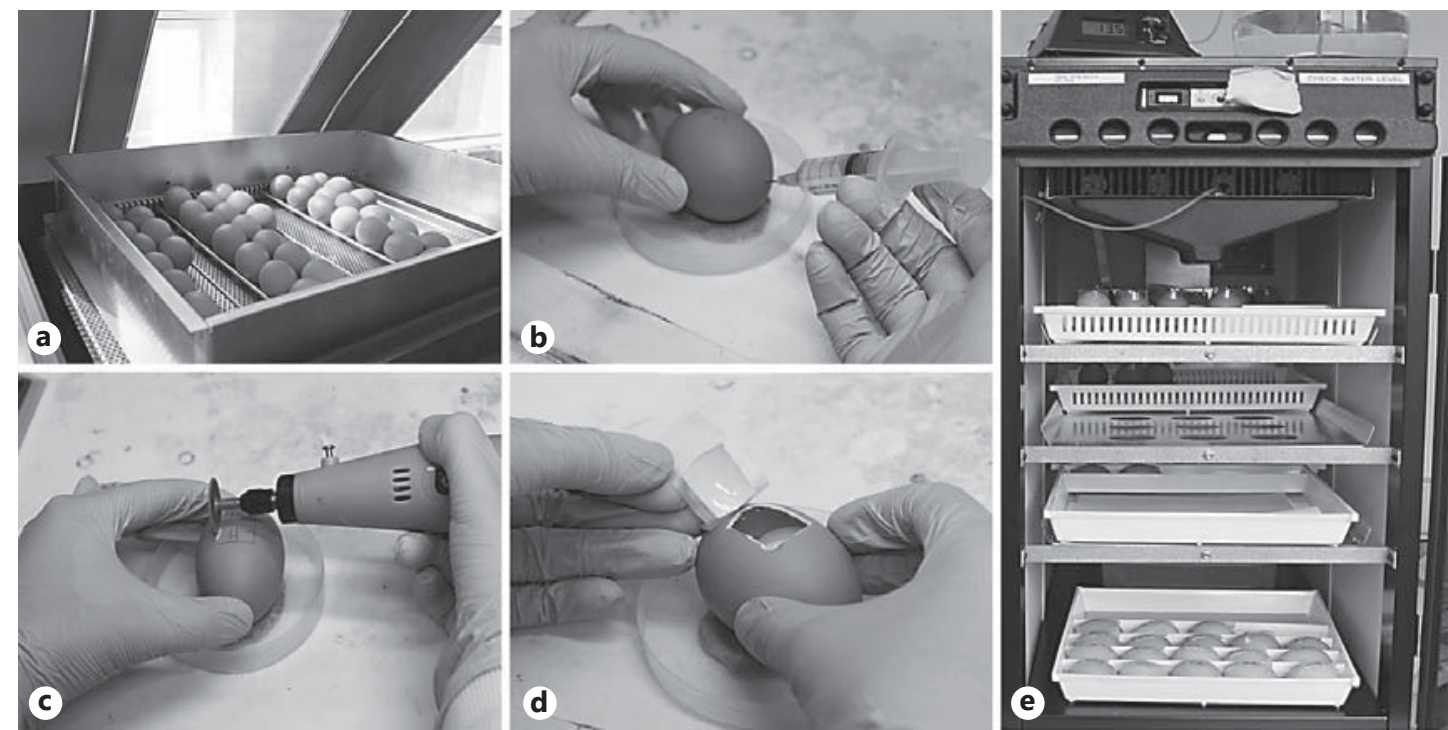

Fig. 1. Overview of the chick embryo egg preparation for CAM assays and injection studies. a Chick embryo eggs are incubated at $37^{\circ} \mathrm{C}$ with regular rotation until processing. $\mathbf{b}$ The CAM is lowered at E3-E4 by removing a small volume (approx. $3 \mathrm{ml}$ ) of albumen with a 5-ml syringe and a 18-gauge needle. c A rotating disc drill is used to cut a small hinged window in the shell. $\mathbf{d}$ The window is used to apply the tumour cells and monitor chick embryo development. It is sealed with clear tape. e The eggs are incubated at $37^{\circ} \mathrm{C}$ and $60 \%$ humidity until final analysis.

surrounding membrane and fixed in 10\% neutral buffered formalin for histological and immunohistochemical examination. Figure 2a-c shows representative images of a CAM nodule formed by 92.1 uveal melanoma cells. The uveal melanoma cells were confirmed by immunohistochemical analysis of MelanA expression (fig. 2f). A high Ki67 proliferation index (fig. $2 \mathrm{~g}$ ) and vascular structures within the tumour mass were also observed. Berube et al. [21] have reported the ability of four other uveal melanoma cell lines, SP6.5, SP8.0, TP31 and $\mathrm{H79}$, to yield vascularised tumour masses on the chick embryo CAM following inoculation of $5 \times 10^{6}$ cells at E10 for 7 days, although no histological analysis of these tumours was reported.

Use of the CAM assay also enables additional steps in the metastatic cascade to be examined. Reports using human fibrosarcoma cells inoculated onto the CAM have not only shown the development of sizeable tumour masses within 7 days, but also that cells from these escape, intravasate into the chick blood vessels and reach distal portions of the CAM and internal organs where they form micro-metastatic foci [22]. Parental 92.1 and OMM1 uveal melanoma cells have to date not been found to escape the tumour mass formed on the CAM during the 7-day incubation period. One possibility is that additional genetic alterations are necessary to increase the metastatic potential of these cells as compared with the parental cell line. The effects of cancer-specific molecular aberrations on tumour mass formation, angiogenesis and dissemination have been examined for a variety of tumour cell types using the CAM assay [23, 24]. Laurent et al. [25] examined the effects of protein tyrosine phosphatase type IV A member 3 (PTP4A3), a predictor of uveal melanoma metastasis, when introduced into the OCM1 uveal melanoma cell line, on tumour cell dissemination using the CAM assay. Although no information was provided regarding the efficiency and size of tumour mass formation on the CAM for the OCM1 cells, an increased invasiveness and migration to the chick embryo femur was reported for the PTP4A3-expressing OCM1 cells as compared with the parental cell line. 

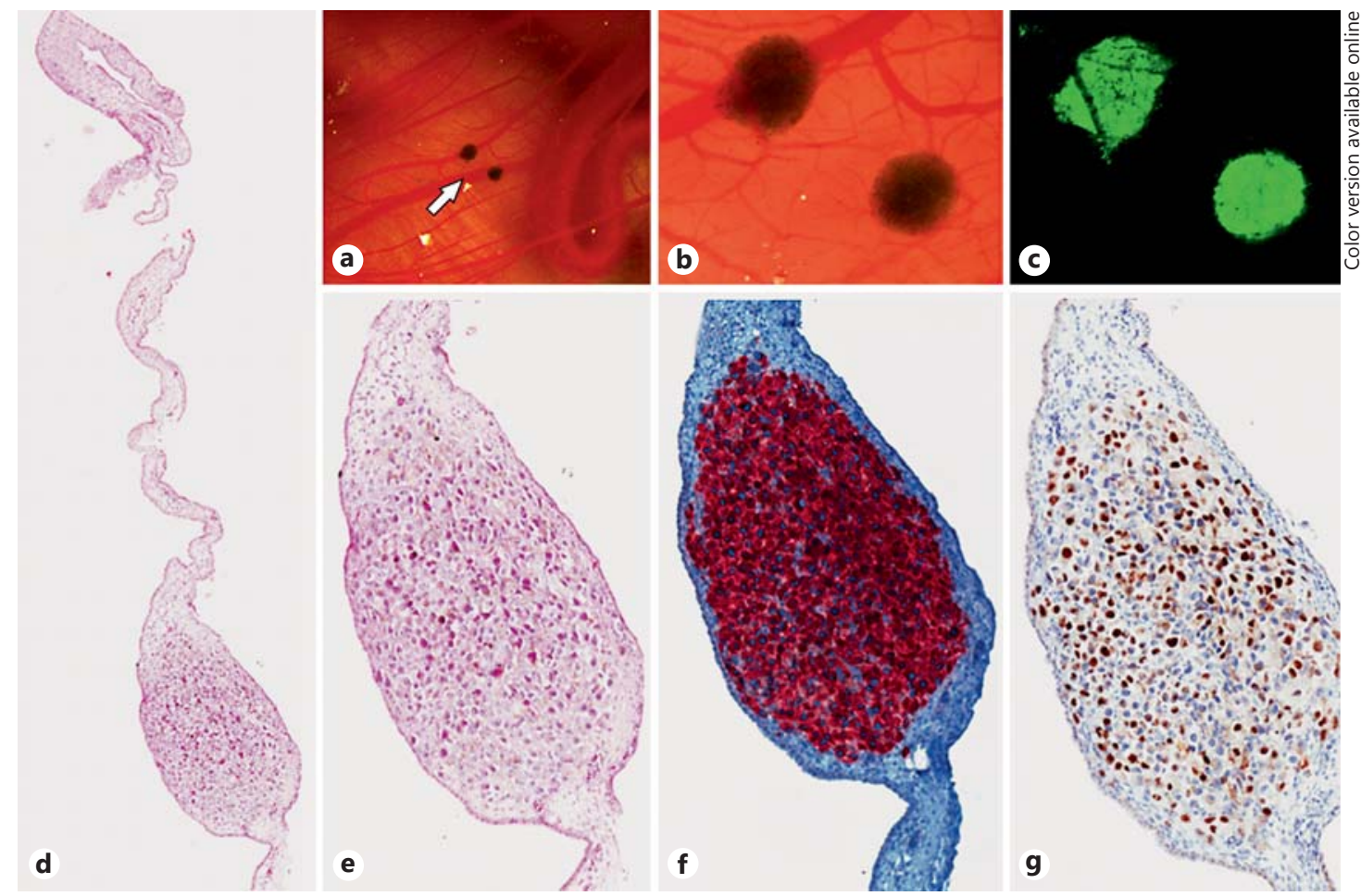

Fig. 2. Macroscopic detection and immunohistochemical analysis of a tumour nodule formed in the CAM assay. 92.1 uveal melanoma cells formed a visible nodule on the CAM 7 days following cell grafting. Low-power brightfield image (a; the arrow shows two pigmented tumour nodules), high-power brightfield image (b) and high-power fluorescence image (c) of the GFP-labelled 92.1 cells. d Haematoxylin \& eosin-stained image of the CAM and cross-section of the tumour nodule (at higher magnification in e). Immunohistochemical analysis shows MelanA positivity (f) and a high Ki67 proliferative index (g) for the 92.1 uveal melanoma cell nodule located within the CAM membrane.

Table 1. Distribution of 92.1 and OMM1 uveal melanoma cells 7 days following injection at E7 in the experimental metastasis model

\begin{tabular}{lll}
\hline Chick tissue & 92.1 & OMM1 \\
\hline Liver & +++ & +++ \\
Eye & +++ & +++ \\
Lungs & - & - \\
Spleen & - & - \\
Heart & + & - \\
Gut & ++ & + \\
Kidneys & ++ & ++ \\
Brain & +++ & ++ \\
\hline
\end{tabular}

An alternative approach in the chick embryo is the experimental metastasis model. In this model, tumour cells are injected directly into the circulation and their ability to extravasate and colonise distant organs is examined. GFP-labelled 92.1 and OMM1 uveal melanoma cells have been injected directly into the chick embryo circulation on E7 and their homing to and colonisation of chick organs examined at E14. Preliminary data suggests that the OMM1 and 92.1 cells are found in the majority of neural crest-derived tissues (table 1), with the highest number and most successful growth occurring in the eye and liver. Both cell lines homed to the chick embryo eye spreading widely within the uveal tract, demonstrating their ability to 
Ocular Oncology

and Pathology

Fig. 3. Homing of GFP-labelled uveal melanoma cells to the chick embryo eye. GFP-labelled 92.1 cells injected on E7 homed to the uveal tract by E14.

Fig. 4. Macroscopic detection and immunohistochemical analysis of uveal melanoma foci in the chick embryo liver. OMM1 cells injected on E7 formed visible tumour foci by E14. A number of foci were visualised following the immunohistochemical detection of MelanA-positive uveal melanoma cells. The tumour foci were detected adjacent to blood vessels in the chick embryo liver.

\begin{tabular}{l|l}
\hline Ocul Oncol Pathol 2015;1:133-140 \\
\hline DOI: 10.1159/000370151 & $\begin{array}{l}\text { (c) 2015 S. Karger AG, Basel } \\
\text { www.karger.com/oop }\end{array}$ \\
\hline
\end{tabular}

Kalirai et al.: Use of the Chick Embryo Model in Uveal Melanoma
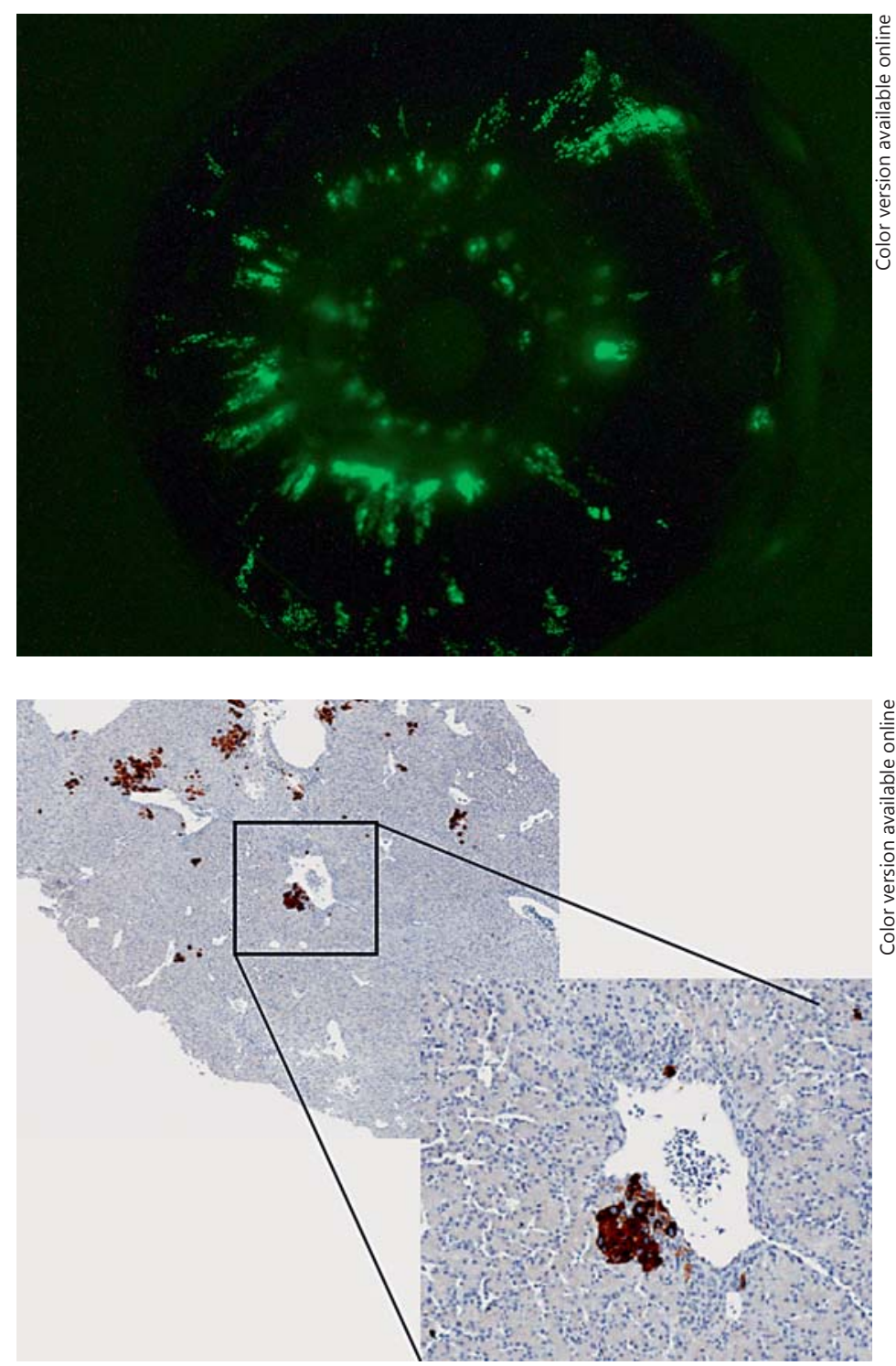

undergo orthotopic growth (fig. 3). Interestingly, this phenomenon has also been seen in the zebrafish model [26]. Histological and immunohistochemical examination of the chick liver demonstrated the presence of micro-metastatic foci of both cell lines in this organ (fig. 4). The tumour cell foci, identified by MelanA immunohistochemistry, were located close to blood vessels and Kupffer cells. This is consistent with the presence of metastatic uveal melanoma cells in the perisinusoidal space of the liver, also known as the space of Disse, in patient specimens [27].

\section{Conclusions and Future Directions}

In summary, the chick embryo model has been used successfully to date in a limited number of studies assessing the ability of uveal melanoma cells to undergo orthotopic growth in the chick eye, form tumour masses on the CAM and undergo dissemination via the chick 
Ocular Oncology

and Pathology
Ocul Oncol Pathol 2015;1:133-140

DOI: 10.1159/000370151

circulation to internal organs. Despite the short time period for tumour growth, it represents a valid model for the analysis of many aspects of uveal melanoma biology. Continued baseline analyses are, however, necessary using a larger panel of well-characterised uveal melanoma cell lines covering the spectrum of genomic and phenotypic alterations observed in patients. This will establish which cell lines efficiently form tumour masses on the CAM. For example, in a study of osteosarcoma cell lines, only three out of eight lines tested demonstrated CAM tumour mass formation [28]. Furthermore, identification of uveal melanoma cell lines with no or limited invasion capacity, following tumour mass formation in the CAM assay, will facilitate the study of genetic alterations and molecules hypothesised to promote invasion. Conversely, the characterisation of uveal melanoma cell lines with enhanced invasive capacity will expedite the identification of therapeutic targets that can prevent metastatic dissemination and/or colonisation at distant sites.

The CAM assay is also an attractive model to rapidly assess the effectiveness of novel candidate therapeutic drugs and inhibitors on tumour growth, an essential component of uveal melanoma translational studies. Hagedorn et al. [11] reported that the treatment of human glioma cells with receptor tyrosine kinase inhibitors inhibited tumour growth in a CAM model, while the chemotherapy agent doxorubicin reduced the growth of human leukaemia cell lines on the CAM [29].

It is also likely, as in current 3D culture systems, that there will be a move from monoculture on the CAM to co-culture systems using, for example, tumour-associated macrophages. A recent study [30] has combined fluorescently-labelled murine mammary carcinoma cells with mammary fibroblasts and grafted these onto the chick embryo CAM to study epithelial/stromal crosstalk. The study demonstrated the ability of stromal cells to drive the migration of mammary tumour cells, again highlighting the utility of this model system. Similarly, the CAM assay has become an attractive tool to follow the fate and visualise microscopically the behaviour of the grafted tumour cells [31]. In vivo microscopy is possible, allowing direct observations of tumour development and escape as well the effects of intravascular or topical application of anticancer drugs [32]. Additionally, in the experimental metastasis model, intravital videomicroscopy of the chorioallantoic microcirculation facilitates investigations of metastasis formation. Epifluorescence and intravital videomicroscopy has been used to identify labelled B16F1 melanoma cells in the CAM and study successive stages of metastasis formation in vivo [33, 34].

In conclusion, the chick embryo models represent valuable, inexpensive and rapid in vivo systems that could complement work performed in rodents to improve our understanding of the metastatic process in uveal melanoma and for the preliminary testing of potential new agents to aid molecular-based cancer therapy.

\section{References}

1 Rous P: A sarcoma of the fowl transmissible by an agent separable from the tumor cells. J Exp Med 1911;13: 397-411.

2 Murphy JB, Rous P: The behavior of chicken sarcoma implanted in the developing embryo. J Exp Med 1912; 15:119-132.

3 Hamburger V, Hamilton HL: A series of normal stages in the development of the chick embryo. 1951. Dev Dyn 1992;195:231-272.

4 Bellairs R, Osmond M: The Atlas of Chick Development. London, Academic Press, 1998.

5 Rous P, Murphy JB: Tumor implantations in the developing embryo. J Am Med Assoc 1911;56:741.

6 Murphy JB: Transplantability of malignant tumors to embryos of a foreign species. J Am Med Assoc 1912;59: 874.

7 Cimpean AM, Ribatti D, Raica M: The chick embryo chorioallantoic membrane as a model to study tumor metastasis. Angiogenesis 2008;11:311-319. 
Kalirai et al.: Use of the Chick Embryo Model in Uveal Melanoma

8 Deryugina EI, Quigley JP: Chick embryo chorioallantoic membrane model systems to study and visualize human tumor cell metastasis. Histochem Cell Biol 2008;130:1119-1130.

9 Kain KH, et al: The chick embryo as an expanding experimental model for cancer and cardiovascular research. Dev Dyn 2014;243:216-228.

10 Palmer TD, Lewis J, Zijlstra A: Quantitative analysis of cancer metastasis using an avian embryo model. J Vis Exp 2011, DOI: 10.3791/2815.

11 Hagedorn M, et al: Accessing key steps of human tumor progression in vivo by using an avian embryo model. Proc Natl Acad Sci USA 2005;102:1643-1648.

12 Conn EM, et al: Comparative analysis of metastasis variants derived from human prostate carcinoma cells: roles in intravasation of VEGF-mediated angiogenesis and uPA-mediated invasion. Am J Pathol 2009;175: 1638-1652.

13 Subauste MC, et al: Evaluation of metastatic and angiogenic potentials of human colon carcinoma cells in chick embryo model systems. Clin Exp Metastasis 2009;26:1033-1047.

14 Fergelot $\mathrm{P}$, et al: The experimental renal cell carcinoma model in the chick embryo. Angiogenesis 2013;16: 181-194.

15 Lokman NA, et al: Chick chorioallantoic membrane (CAM) assay as an in vivo model to study the effect of newly identified molecules on ovarian cancer invasion and metastasis. Int J Mol Sci 2012;13:9959-9970.

16 Wan J, et al: HIF-1alpha effects on angiogenic potential in human small cell lung carcinoma. J Exp Clin Cancer Res 2011;30:77.

17 Luyten GPM, et al: A chicken embryo model to study the growth of human uveal melanoma. Biochem Biophys Res Commun 1993;192:22-29.

18 Busch C, Krochmann J, Drews U: The chick embryo as an experimental system for melanoma cell invasion. PLoS One 2013;8:e53970.

19 Luyten GP, et al: Establishment and characterization of primary and metastatic uveal melanoma cell lines. Int J Cancer 1996;66:380-387.

20 De Waard-Siebinga I, et al: Establishment and characterization of an uveal-melanoma cell line. Int J Cancer 1995;62:155-161.

21 Berube M, et al: MMP-2 expression in uveal melanoma: differential activation status dictated by the cellular environment. Mol Vis 2005;11:1101-1111.

22 Deryugina EI, et al: Unexpected effect of matrix metalloproteinase down-regulation on vascular intravasation and metastasis of human fibrosarcoma cells selected in vivo for high rates of dissemination. Cancer Res 2005; 65:10959-10969.

23 Bader AG, Kang S, Vogt PK: Cancer-specific mutations in PIK3CA are oncogenic in vivo. Proc Natl Acad Sci USA 2006;103:1475-1479.

24 Carter R, et al: Exploitation of chick embryo environments to reprogram MYCN-amplified neuroblastoma cells to a benign phenotype, lacking detectable MYCN expression. Oncogenesis 2012;1:e24.

25 Laurent C, et al: High PTP4A3 phosphatase expression correlates with metastatic risk in uveal melanoma patients. Cancer Res 2011;71:666-674.

26 van der Ent W, et al: Embryonic zebrafish: different phenotypes after injection of human uveal melanoma cells. Ocul Oncol Pathol 2015;1:170-181.

27 Shahidipour $\mathrm{H}$, et al: Chick embryo model systems to study uveal melanoma metastasis. Orlando, The Association for Research in Vision and Opthalmology, 2014.

28 Balke M, et al: Morphologic characterization of osteosarcoma growth on the chick chorioallantoic membrane. BMC Res Notes 2010;3:58.

29 Taizi M, et al: A novel and rapid in vivo system for testing therapeutics on human leukemias. Exp Hematol 2006;34:1698-1708.

30 Matise LA, et al: Lack of transforming growth factor-beta signaling promotes collective cancer cell invasion through tumor-stromal crosstalk. Breast Cancer Res 2012;14:R98.

31 Fein MR, Egeblad M: Caught in the act: revealing the metastatic process by live imaging. Dis Model Mech 2013; 6:580-593.

32 Zijlstra A, et al: The inhibition of tumor cell intravasation and subsequent metastasis via regulation of in vivo tumor cell motility by the tetraspanin CD151. Cancer Cell 2008;13:221-234.

33 Chambers AF, et al: Early steps in hematogenous metastasis of B16F1 melanoma cells in chick embryos studied by high-resolution intravital videomicroscopy. J Natl Cancer Inst 1992;84:797-803.

34 MacDonald IC, et al: Intravital videomicroscopy of the chorioallantoic microcirculation: a model system for studying metastasis. Microvasc Res 1992;44:185-199. 


\section{Erratum}

In the article by Kalirai $\mathrm{H}$, Shahidipour $\mathrm{H}$, Coupland $\mathrm{SE}$ and Luyten GPM entitled 'Use of the Chick Embryo Model in Uveal Melanoma' [Ocul Oncol Pathol 2015;1:133-140, DOI: $10.1159 / 000370151]$, the following author needs to be added as the third author:

\section{Diana Moss}

Department of Cellular and Molecular Physiology, Institute of Translational Medicine, University of Liverpool, Liverpool, UK 